VOL. $20(1979), 301-312$.

\title{
On geometric duality for state-constrained control problems
}

\section{T.R. Jefferson and C.H. Scott}

\begin{abstract}
For convex optimal control problems without explicit pure state constraints, the structure of dual problems is now well known. However, when these constraints are present and active, the theory of duality is not highly developed. The major difficulty is that the dual variables are not absolutely continuous functions as a result of singularities when the state trajectory hits a state constraint. In this paper we recognize this difficulty by formulating the dual probram in the space of measurable functions. A strong duality theorem is derived. This pairs a primal state constrained convex optimal control problem with a dual convex control problem that is unconstrained with respect to state constraints. In this sense, the dual problem is computationally more attractive than the primal.
\end{abstract}

\section{Introduction}

Perhaps the most powerful concept associated with modern optimization theory is that of duality by which one can replace an optimization problem by an equivalent, but structurally different problem. This has proved immensely profitable for mathematical programs both in the computational and interpretational sense. Currently much of this work is being generalised from finite dimensional spaces associated with mathematical programing to various function spaces [2]. As such a mathematical setting directly embraces the field of optimal control theory, it is natural that there is now considerable interest in duality for optimal control problems.

Received 30 March 1979. 
Previous developments have used both lagrangian [1], [3], and conjugate function methods [4], [5], [6], [8], [11], to derive duals to control problems. Generally speaking, conjugate function approaches are preferable to lagrangian methods since they completely separate primal and dual variables and result in an independent dual program. However, these methods have not, as yet, handled pure state constraints explicitly.

Here we develop a dual formulation for an optimal control problem with explicit constraints on the state variables. Specifically we use an extension of generalised geometric programming [7] to function spaces. Technical complications arise since explicit state constraints in the primal induce singularities in the dual (costate) variables. Hence the dual must be formulated in a very general function space; the space of measurable functions. We obtain a dual which is unconstrained as far as explicit constraints are concerned. This is of computational value in the development of algorithms.

\section{The control formulation}

We consider an optimal control problem of the form

(I) (A) minimize $\left(\int_{0}^{T} f_{1}\left(t, x_{1}(t), u_{1}(t)\right) d t+L\left(x_{0}(0), x_{0}(T)\right)\right)$

subject to explicit state constraints,

$$
f_{i}\left(t, x_{i}(t)\right) \leq 0 \text {, almost everywhere on }[0, T], i \in I_{1},
$$

explicit control constraints,

$$
f_{i}\left(t, u_{i}(t)\right) \leq 0 \text {, almost everywhere on }[0, T], i \in I_{2},
$$

explicit mixed constraints,

(4) $f_{i}\left(t, x_{i}(t), u_{i}(t)\right) \leq 0$, almost everywhere on $[0, T], i \in I_{3}$, implicit constraints,

$$
x_{1} \in C_{1}, u_{1} \in U_{1} \text {, }
$$

and a subspace condition, 
(6) $x_{0}=x_{i}, u_{0}=u_{i}$, almost everywhere on $[0, T], i \in \underset{k=1}{U} I_{k}$, (7) $\dot{x}_{0}(t)=A(t) x_{0}(t)+u_{0}(t)$, almost everywhere on $[0, T]$.

The minimization is taken over the states $x_{i}:[0, T] \rightarrow R^{n}$, absolutely continuous functions for any $i$ and controls $u_{i}:[0, T] \rightarrow R^{n}$, measurable for any $i \cdot x_{1}$ is restricted to the set of functions $C_{1}$ which is closed and convex; $u_{1}$ is restricted to the closed and convex set of functions $U_{1}$. The $f_{i}(t, \cdot, \cdot)$ and $f_{i}(t, \cdot)$ are closed convex functions. $l$ is a closed convex function of the initial and terminal states.

For program A to be well defined, we require that:

(i) $f_{i}(t, \cdot), f_{i}(t, \cdot, \cdot)$ are normal integrands in the sense of Rockafellar [9];

(ii) $A(t) x_{0}(t)$ is a normal integrand;

(iii) there exists a finite bounded solution to (A).

The system dynamics (7) may be solved and yield

$$
x_{0}(t)=\Phi(t, 0) x_{0}(0)+\int_{0}^{t} \Phi(t, \tau) u_{0}(\tau) d \tau,
$$

where $\Phi$ is the transition matrix and satisfies

$$
\dot{\Phi}=A(t) \Phi(t, \tau)
$$

with $\Phi(\tau, \tau)=I$.

With the program, as stated, we can commence the development of a duality theory which is suitable for handing state constraints. In the following section, we will develop duality in this context. The resulting conjugacy theory will be applied to program A to develop program $B$, its geometric dual, in Section 4. 


\section{Conjugacy}

In order to handle state constraints, we require the most general pairing of dual spaces; that is, function spaces with measures. For our purposes, we will decompose the measure into two parts: absolutely continuous and singular. Thus the function $x(t)$ is paired with $y_{a}(t)$ and $y_{s}(d t)$ so that the inner product is

$$
\langle x, y\rangle=\int_{0}^{T} x(t) y_{a}(t) d t+\int_{0}^{T} x(t) y_{s}(d t)
$$

It has been shown [10], that $y_{s}$ is zero in the absence of state constraints. However, as we propose to deal with state constraints, it is necessary to develop conjugacy in full generality.

DEFINITION 1. The conjugate transform of a functional

$\int_{0}^{T} f(t, x, u) d t$ defined on $x \in C$ and $\left.u \in U, \quad\left[\int f d t, C \times U\right]\right)$ is $[G, D]$ and is defined by

$$
\begin{aligned}
G(y, v) & =\int_{0}^{T} g\left(t, y_{a}, v_{a}\right) d t+\int_{0}^{T} s\left(t, y_{s}(d t), v_{s}(d t)\right) \\
& =\sup _{\substack{x \in C \\
u \in U}}\left\{(x, y)+\langle u, v\rangle-\int_{0}^{T} f(t, x, u) d t\right\},
\end{aligned}
$$

where $g$ and $s$ are conjugate transforms of the integrand at time $t([f, C(t) \times U(t)])$ and are its absolutely continuous and singular parts respectively. In particular

$$
\begin{gathered}
g\left(t, y_{a}, v_{a}\right)=\sup _{\substack{x(t) \in C(t) \\
u(t) \in U(t)}}\left\{\left\langle x(t), y_{a}(t)\right\rangle+\left\langle u(t), v_{a}(t)\right\rangle\right. \\
s\left(t, y_{s}(d t), v_{s}(d t)\right)=\sup _{\substack{x(t) \in C(t) \\
u(t) \in U(t)}}\left\{\left\langle x(t), y_{s}(d t)\right)+\left\langle u(t), v_{s}(d t)\right\rangle\right\},
\end{gathered}
$$

where $(\cdot, \cdot)$ denotes the usual inner product, 


$$
D=\left\{(y, v) \mid \sup _{\substack{x \in C \\ u \in U}}\left\{(x, y)+(u, v)-\int_{0}^{T} f(t, x, u) d t\right\}<\infty\right\} .
$$

Further $g$ and $s$ are defined on $D_{a}(t)$ and $D_{s}(t)$ respectively, where

$$
\begin{aligned}
& D_{a}(t)=\left\{\left(y_{a}(t), v_{a}(t)\right) \mid \sup _{\substack{x(t) \in C(t) \\
u(t) \in U(t)}}\left\{\left\langle x(t), y_{a}(t)\right\rangle+\left\langle u(t), v_{a}(t)\right\rangle\right.\right. \\
& D_{s}(t)=\left\{\left(y_{s}(d t), v_{s}(d t)\right) \mid \sup _{\substack{x(t) \in C(t) \\
u(t) \in U(t)}}\left\{\left\langle x(t), y_{s}(d t)\right\rangle+\left\langle u(t), v_{s}(d t)\right\rangle\right\}<\infty\right\} . \\
& \text { Hence } D=\sin _{t \in[0, T]}\left(D_{a}(t) \times D_{s}(t)\right) .
\end{aligned}
$$

In the case of constraints such as

$$
f(t, x, u) \leq 0 \text {, almost everywhere on }[0, T],
$$

the conjugate transform of $[0, f(t, x(t), u(t)) \leq 0]$ is the positive homogeneous extension of the conjugate transform

$$
g\left(t, y_{a}(t), v_{a}(t)\right) d t+s\left(t, y_{s}(d t), v_{s}(d t)\right) \text {. }
$$

DEFINITION 2. The positive homogeneous extension of $g\left(t, y_{a}(t), v_{a}(t)\right)$ is $g^{+}\left(t, y_{a}(t), v_{a}(t) ; \lambda_{a}(t)\right)$ and is given by $g^{+}\left(t, y_{a}(t), v_{a}(t) ; \lambda_{a}(t)\right)=\left\{\begin{array}{l}\sup _{x(t) \in C(t)}\left\{\left\langle x(t), y_{a}(t)\right\rangle+\left\langle u(t), v_{a}(t)\right\rangle\right\} \\ u(t) \in U(t) \\ \quad \text { if } \lambda_{a}(t)=0, \\ \lambda_{a}(t) g\left(t, y_{a}(t) / \lambda_{a}(t), v_{a}(t) / \lambda_{a}(t)\right) \\ \text { if } \lambda_{a}(t)>0 .\end{array}\right.$

DEFINITION 3 . The positive homogeneous extension of $s\left(t, y_{s}(d t), v_{s}(d t)\right)$ is $s^{+}\left(t, y_{s}(d t), v_{s}(d t) ; \lambda_{s}(d t)\right)$ and is given by 
$s^{+}\left(t, y_{s}(d t), v_{s}(d t) ; \lambda_{s}(d t)\right)= \begin{cases}\sup _{x(t) \in C(t)}\left\{\left(x(t), y_{s}(d t)\right)+\left(u(t), v_{s}(d t)\right\rangle\right\} \\ u(t) \in U(t) & \text { if } \lambda_{s}(d t)=0, \\ \lambda_{s}(d t) s\left(t, y_{s}(d t) / \lambda_{s}(d t),\right. & \left.v_{s}(d t) / \lambda_{s}(d t)\right) \\ & \text { if } \lambda_{s}(d t)>0 .\end{cases}$

Here $\langle\cdot, \cdot\rangle$ represents the usual inner product; $y_{\mathcal{S}}(d t) / \lambda_{\mathcal{S}}(d t)$ and $v_{s}(d t) / \lambda_{s}(d t)$ are the Radon-Nikodym derivatives. Both $s$ and $s^{+}$are well defined from the original definition of $s ; g^{+}$and $s^{+}$are defined on $D_{a}^{+}(t)$ and $D_{s}^{+}(t)$ where,

$$
\begin{aligned}
& D_{a}^{+}(t)=\left\{\left(y_{a}(t), v_{a}(t) ; \lambda_{a}(t)\right) \mid \sup _{\substack{x(t) \in C(t) \\
u(t) \in U(t)}}\left\{\left(x(t), y_{a}(t)\right\rangle\right.\right. \\
& \left.\left.+\left(u(t), v_{a}(t)\right)\right\}<\infty \text { for } \lambda_{a}(t)=0\right\} \\
& \quad \cup\left\{\left(y_{a}(t), v_{a}(t) ; \lambda_{a}(t)\right) \mid\left(y_{a}(t) / \lambda_{a}(t), v_{a}(t) / \lambda_{a}(t)\right) \in D_{a}(t), \lambda_{a}(t)>0\right\}
\end{aligned}
$$

and

$$
\begin{aligned}
& D_{s}^{+}(t)=\left\{\left(y_{s}(d t), v_{s}(d t) ; \lambda_{s}(d t)\right) \mid \sup _{x(t) \in C(t)}\left\{\left(x(t), y_{s}(d t)\right\}\right.\right. \\
& u(t) \in U(t) \\
& \left.+\left\langle u(t), v_{s}(d t)\right\rangle\right\}<\infty \text { for } \lambda_{s}(d t)=0 \text { ) } \\
& u\left\{\left(u_{s}(d t), v_{s}(d t) ; \lambda_{s}(d t)\right) \mid\left(y_{s}(d t) / \lambda_{s}(d t), v_{s}(d t) / \lambda_{s}(d t)\right) \in D_{s}(t)\right. \text {, } \\
& \lambda_{s}(d t)>\text { 아. }
\end{aligned}
$$

Thus the conjugate transform of $|0, f(t) \leq 0, t \in[0, T]|$ is $\left[G^{+}(y, v ; \lambda), D^{+}\right]$and is defined by

$$
\begin{aligned}
G^{+}(y, v ; \lambda)=\int_{0}^{T} g^{+}\left(t, y_{a}(t), v_{a}(t) ; \lambda_{a}(t)\right) d t & \\
& +\int_{0}^{T} s^{+}\left(t, y_{s}(d t), v_{s}(d t) ; \lambda_{s}(d t)\right)
\end{aligned}
$$

and $D^{+}=\underset{t \in[0, T]}{\times}\left(D_{a}^{+}(t) \times D_{s}^{+}(t)\right)$.

As a consequence of the definition of the conjugate transform, we have the following conjugate inequality. For $x \in \mathcal{C}$ and $u \in U$, 
(11) $\int_{0}^{T} f(t, x, u) d t+\int_{0}^{T} g\left(t, y_{a}, v_{a}\right) d t+\int_{0}^{T} s\left(t, y_{s}(d t), v_{s}(d t)\right)$

$$
\geq\langle x, y\rangle+\langle u, v\rangle \text {. }
$$

For the particular case of the positive homogeneous extension, the conjugate inequality takes the form

$$
\begin{aligned}
\int_{0}^{T} g^{+}\left(t, y_{\alpha}(t), v_{a}(t) ; \lambda_{\alpha}(t)\right) d t+\int_{0}^{T} s^{+}\left(t, y_{s}(d t)\right. & \left.,{ }_{s}(d t) ; \lambda_{s}(d t)\right) \\
& \geq\langle x, y\rangle+\langle u, v\rangle,
\end{aligned}
$$

for $x \in C, u \in U, f(t, x, u) \leq 0$, almost everywhere on $[0, T]$, and $(y, v ; \lambda) \in D^{+}$.

We are interested, of course, in the inequality holding at equality since in this case there is no duality gap. In order to study this, we require the concept of subvariation.

DEFINITION 4. The subvariation of a functional $\int f(t, x, u) d t$ is the set $\partial \int f(t, x, u) d t$ where

$$
\begin{aligned}
& \partial \int f(t, x, u) d t=\left\{(y, v) \mid \int f(t, x, u) d t+(\hat{x}-x, y\rangle+\langle\hat{u}-u, v\rangle\right. \\
&\left.\leq \int f(t, \hat{x}, \hat{u}) d t, \text { for all }(\hat{x}, \hat{u}) \in C \times U\right\} .
\end{aligned}
$$

Hence, by construction, (11) holds at equality if and only if $(y, v) \in \partial \int f(t, x, u) d t$. For the positive homogeneous extension, we have two cases to consider. If $\lambda>0$, then (12) holds at equality if and only if $(y / \lambda, v / \lambda) \in \partial \int f(t, x, u) d t$. This is a consequence of the conjugate inequality for convex functionals. We now consider the case of $\lambda_{a}(t)=0$, which implies that $\left(y_{a}(t), v_{a}(t)\right)=(0,0)$, and $\lambda_{s}(d t)=0$, which implies that $\left(y_{s}(d t), v_{s}(d t)\right)=(0,0)$. This is the case when $(x, u)$ are defined over the whole space, which is the case for constraints (2), (3), and (4) in (A). With the convention that $\lambda \partial \int f(t, x, u) d t$ means that the scalar multiplier $\lambda_{a}$ multiplies the absolutely continuous 
part of $\partial \int f(t, x, u) d t$ and $\lambda_{s}$ multiplies its singular part, (12) holds at equality if and only if $(y, v) \in \lambda \partial \int f(t, x, u) d t$.

\section{Duality}

The dual to (A) is the following optimal control problem

(B) minimize $\int_{0}^{T} g_{I}\left(t, y_{1 a}, v_{1 a}\right) d t+\sum_{i \in I_{I}} \int g_{i}^{+}\left(t, y_{i a} ; \lambda_{i a}\right) d t$

$$
\begin{aligned}
& +\sum_{i \in I_{2}} \int g_{i}^{+}\left(t, v_{i a} ; \lambda_{i a}\right) d t+\sum_{i \in I_{3}} \int g_{i}^{+}\left(t, y_{i a}, v_{i a} ; \lambda_{i a}\right) d t \\
& +m\left(y_{0}(0), y_{0}(T)\right)+\int_{0}^{T} s_{1}\left(t, y_{1 s}(d t)\right)+\sum_{i \in I_{1}} \int_{0}^{T} s_{i}^{+}\left(t, y_{i s}(d t) ; \lambda_{i s}(d t)\right)
\end{aligned}
$$

subject to implicit constraints:

$$
\begin{aligned}
& \left(y_{0}(0), y_{0}(T)\right) \in M, \\
& \left(y_{1}, v_{1}\right) \in D_{1}, \\
& \left(y_{i} ; \lambda_{i}\right) \in D_{i}^{+}, \quad i \in I_{1}, \\
& \left(v_{i} ; \lambda_{i}\right) \in D_{i}^{+}, \quad i \in I_{2}, \\
& \left(y_{i}, v_{i} ; \lambda_{i}\right) \in D_{i}^{+}, \quad i \in I_{3},
\end{aligned}
$$

and subspace

$$
\begin{aligned}
\int_{0}^{T} \Phi^{*}(t, 0)\left\{_{i \in\{0, I\} \cup I_{1} \cup I_{2} \cup I_{3}} y_{i a}(t)\right) d t & \\
& +\int_{0}^{T} \Phi^{*}(t, 0)\left(\sum_{i \in\{i\} \cup I_{1}} y_{i s}(d t)\right)=0,
\end{aligned}
$$

and 


$$
\begin{aligned}
\sum_{i \in\{I\} \cup I_{2} \cup I_{3}} v_{i}(t)=-\int_{t}^{T} \Phi^{*}(\tau, t)\left(\sum_{i \in\{0, I\} \cup I_{1} u I_{2} u I_{3}} y_{i a}(\tau)\right) d \tau & \\
& \left.-\int_{t}^{T} \Phi^{*}(\tau, t) \sum_{i \in\{I\} \cup I_{1}} y_{i s}(d \tau)\right),
\end{aligned}
$$

where $\Phi^{*}$ denotes the adjoint of $\Phi$.

We note that singular parts only appear in the costate variables $i \in\{1\} \cup I_{1}$ and are associated with the implicit and explicit primal pure state constraints. The dual subspace is the orthogonal complimentary subspace of the primal subspace (6) and (7); $m$ is the conjugate transform of 2 and is defined on the convex set $M . \int g_{1} d t+\int s_{1}(d t)$ is the conjugate transform of $\int f_{1} d t . \int g_{i}^{+} d t+\int s_{i}^{+}(d t), i \in I_{1}$, is the positive homogeneous extension of the conjugate transform of $\int f_{i} d t$, $i \in I \cdot \int g_{i}^{+} d t, i \in I_{2} \cup I_{3}$, is the absolutely continuous part of the positive homogeneous extension of the conjugate transform of $\int f_{i} d t$, $i \in I_{2} \cup I_{3}$. The implicit constraints derive from the associated conjugate transforms.

At the optimality, we have for feasible primal and dual trajectories that

$$
\begin{gathered}
y_{0} \in \partial z\left(x_{0}\right), \\
y_{1} \in \partial \int f_{1}\left(t, x_{1}, u_{1}\right) d t, \\
y_{i} \in \lambda_{i} \partial \int f_{i}\left(t, x_{i}\right) d t, i \in I_{1}, \\
v_{i} \in \lambda_{i} \int f_{i}\left(t, u_{i}\right) d t, i \in I_{2}, \\
\left(y_{i}, v_{i}\right) \in \lambda_{i} \partial \int f_{i}\left(t, x_{i}, u_{i}\right) d t, i \in I_{3} .
\end{gathered}
$$

The results given in this section are proved in Theorem 1 which follows. 
THEOREM 1. Given that Programs $\mathrm{A}$ and $\mathrm{B}$ are both consistent and the functionals have the properties stated in section 2, then for feasible solutions $(x, u)$ and $(y, v, \lambda)$ of Programs A and B respectively, we have that $(x, u),(y, v, \lambda)$ satisfy the optimality conditions if and only if $(x, u)$ and $(y, v, \lambda)$ are optimal for Programs $\mathrm{A}$ and $\mathrm{B}$ respectively.

In either case, the primal and dual objective functions sum to zero.

Proof. Assume first that the optimal points for Programs $A$ and $B$ are $\left(x^{*}, u^{*}\right)$ and $\left(y^{*}, v^{*}, \lambda^{*}\right)$ respectively. Let $\left(y^{\prime}, v^{\prime}, \lambda^{\prime}\right)$ satisfy the optimality conditions with respect to $\left(x^{*}, u^{*}\right)$; that is,

$$
\begin{gathered}
y_{0}^{\prime} \in \partial z\left(x_{0}^{*}\right), \\
y_{1}^{\prime} \in \partial \int f_{1}\left(t, x_{1}^{*}, u_{1}^{*}\right) d t, \\
y_{i}^{\prime} \in \lambda_{i}^{\prime} \partial \int f_{i}\left(t, x_{i}^{*}\right) d t, i \in I_{1}, \\
v_{i}^{\prime} \in \lambda_{i}^{\prime} \partial \int f_{i}\left(t, u_{i}^{*}\right), i \in I_{2}, \\
\left(y_{i}^{\prime}, v_{i}^{\prime}\right) \in \lambda_{i}^{\prime} \partial \int f_{i}\left(t, x_{i}^{*}, u_{i}^{*}\right), i \in I_{3} .
\end{gathered}
$$

We condense these results to

$$
\left(y^{\prime}, v^{\prime}, \lambda^{\prime}\right) \in \partial F\left(x^{*}, u^{*}\right) \text {. }
$$

Since the functionals are closed and convex, the primal and dual objective functions sum to $\left\langle x^{*}, y^{\prime}\right\rangle+\left\langle u^{*}, v^{\prime}\right\rangle$. We define $x$ to be the subspace associated with $(6),(7)$ of Program A. If $\partial F\left(x^{*}, u^{*}\right) \cap \chi^{\star} \neq \emptyset$, choose $\left(y^{\prime}, v^{\prime}, \lambda^{\prime}\right)$ to be in this intersection. Then $\left\langle x^{*}, y^{\prime}\right\rangle+\left(u^{*}, v^{\prime}\right)=0$. If $\left(y^{*}, v^{*}, \lambda^{*}\right)$ is optimal for Program B, it must belong to $\partial F\left(x^{*}, u^{*}\right) \cap \chi^{\perp}$ since otherwise it would be either infeasible or non-optimal.

We now prove $\partial F\left(x^{*}, u^{*}\right) \cap \chi^{\perp}$ to be non empty by contradiction. Suppose $\partial F\left(x^{*}, u^{*}\right) \cap \chi^{1}=\varnothing$. Let $P\left(x^{*}, u^{*}\right)$ be the closed convex cone of feasible directions at $\left(x^{*}, u^{*}\right)$. Now $\partial F^{*}\left(x^{*}, u^{*}\right) \cap P\left(x^{*}, u^{*}\right)$ is a compact convex set and $X^{\perp}$ is a subspace.

By the Hahn-Banach Theorem, there exists a function $(\hat{x}, \hat{u})$ such that

$$
\langle\hat{x}, y\rangle+(\hat{u}, v\rangle=0 \text { for }(y, v, \lambda) \in \mathrm{x}^{\perp} \text {, }
$$


and

$$
\langle\hat{x}, y\rangle+(\hat{u}, v\rangle<0 \text { for }(y, v, \lambda) \in \partial F\left(x^{*}, u^{*}\right) \cap P\left(x^{*}, u^{*}\right) .
$$

This implies that $\left(x^{*}+\alpha \hat{x}, u^{*}+\alpha \hat{u}\right) \in X$ and the objective function of the primal decreases for some positive value of $\alpha$. This contradicts the optimality of $\left(x^{*}, u^{*}\right)$.

Suppose now that $\left(x^{*}, u^{*}\right)$ and $\left(y^{*}, v^{*}, \lambda^{*}\right)$ satisfy feasibility and the optimality conditions. The primal and dual objective functions sum to zero as a direct consequence of feasibility and the conditions for equality of the conjugate inequality. Optimality is guaranteed by the fact that the negative of one objective function provides a lower bound on the value of the other and the fact that they sum to zero. Thus $\left(x^{*}, u^{*}\right)$ and $\left(y^{*}, v^{*}, \lambda^{*}\right)$ are optimal.

\section{References}

[1] Viorel Barbu, "On the control problem of Bloza in Hilbert spaces", SIAM J. Control 13 (1975), 1062-1076.

[2] Ivar Ekeland, Roger Teman, Convex analysis and variational problems (Studies in Mathematics and its Applications, 1. North-Holland, Amsterdam, Oxford; American Elsevier, New York; 1976).

[3] William W. Hager and Sanjoy K. Mitter, "Lagrange duality theory for convex control problems", SIAM J. Control Optim. 14 (1976), 843-856.

[4] W. Heins and S.K. Mitter, "Conjugate convex functions, duality, and optimal control problems I: systems governed by ordinary differential equations", Inform. Sci. 2 (1970), 2ll-243.

[5] T.R. Jefferson and C.H. Scott, "Generalised geometric programming applied to problems of optimal control. I: theory", J. Optim. Theory App 2.26 (1978), 117-129.

[6] John D. Pearson, "Reciprocity and duality in control programing problems", J. Math. Anal. Appl. 10 (1965), 388-408.

[7] Elmor L. Peterson, "Geometric programming", SIAM Rev. 18 (1976), $1-51$. 
[8] R.T. Rockafellar, "Conjugate convex functions in optimal control and the calculus of variations", J. Math. Anal. Appl. 32 (1970), $174-222$.

[9] R.T. Rockafellar, "Integrals which are convex functionals, II", Pacific J. Math. 39 (1971), 439-469.

[10] R. Tyrrell Rockafellar, "State constraints in convex control problems of Bolza", SIAM J. Control 10 (1972), 691-715.

[11] R.B. Vinter, "Application of duality theory to a class of composite cost control problems", J. Optim. Theory Appl. 13 (1974), $436-460$.

School of Mechanical and Industrial Engineering,

University of New South Wales,

Kensington,

New South wales. 